\title{
Spontaneous energy-barrier formation in entropy-driven glassy dynamics
}

\author{
Chiara Cammarota* \\ Sapienza, University of Rome P.le Aldo Moro 2, I-00185 Rome, Italy
}

Enzo Marinari ${ }^{\dagger}$

Sapienza, University of Rome and IPCF-CNR, UOS Rome P.le Aldo Moro 2, I-00185 Rome, Italy

(Received 8 October 2014; revised manuscript received 13 May 2015; published 9 July 2015)

\begin{abstract}
The description of activated relaxation of glassy systems in the multidimensional configurational space is a long-standing open problem. We develop a phenomenological description of the out-of-equilibrium dynamics of a model with a rough potential energy landscape and we analyze it both numerically and analytically. The model provides an example of dynamics where typical relaxation channels go over finite-potential energy barriers despite the presence of less-energy-demanding escaping paths in configurational space; we expect this phenomenon to be also relevant in the thermally activated regime of realistic models of glass-formers. In this case, we found that typical dynamical paths episodically reach an high-fixed-threshold energy, unexpectedly giving rise to a simple thermally activated aging phenomenology. In order to unveil this peculiar aging behavior, we introduce a novel description of the dynamics in terms of spontaneously emerging dynamical basins.
\end{abstract}

DOI: 10.1103/PhysRevE.92.010301

PACS number(s): 81.40.Cd, 05.10.Ln, 05.45.-a, 64.70.pm

Introduction. Glass formation manifests itself as a dynamic phenomenon. Below a crossover temperature $T_{g}$, supercooled systems (characterized by aperiodic disordered patterns in the metastable liquid phase) run out of equilibrium on the timescale of experimental observations [1]. When approaching from above the temperature of the glass-formation crossover, the growth of the relaxation timescale is faster than an Arrhenius behavior, $\tau \sim \exp (\Delta / T)$, where $\Delta$ is a fixed activation barrier and $T$ is the temperature. This observation originally suggested that the progressively slower relaxation dynamics would be the result of activated processes involving increasing activation barriers $\Delta(T)$ when $T$ decreases [2].

Providing a quantitative description of glassy dynamics in terms of activated processes is still an open problem. A long theoretical effort started with the definition of simple but interesting models for thermal activation called trap models [3,4]. New results [5-9] recently confirmed the broad interest of the trap-model paradigm for the description of realistic activated dynamics and inspired the present work. A number of alternative pictures have been proposed in the literature, ranging from a microscopic dynamical theory of nonactivated relaxation [10], called mode-coupling theory, and other attempts toward the formulation of a purely dynamical picture free from any landscape influence $[11,12]$, to a peculiar thermodynamic theory of the slow dynamics, the random first-order transition (RFOT) theory [13-17], whose main ingredient is the rough (complex) structure of the potential energy landscape (PEL) in a multidimensional configurational space. In these approaches, the large dimensionality of the configurational space can play different roles, both in the equilibrium and out-of-equilibrium dynamics. On the one side, it is possible to show that the activated relaxation through a barrier of high $\Delta$ in any direction of a large-dimensional space should occur on average on a timescale smaller than

\footnotetext{
*chiara.cammarota@roma1.infn.it

†enzo.marinari@uniroma1.it
}

the expected Arrhenius $\tau \sim \exp (\Delta / T)$ [18]. The entropy of possible escaping directions produces as an effect the lowering of the activation barrier. On the other side, for nonactivated processes, the dynamics is slowed down by the presence of an overwhelming number of directions in the configurational space that do not allow the system to relax till one of the rare escaping channels is found (entropic bottleneck effect) [19-21].

These two mechanisms have been investigated separately, respectively, within the thermodynamic-influenced RFOT picture (where a Hamiltonian defines a rough equilibrium landscape) and the nonactivated purely dynamical approach (based on kinetically constrained models, where thermodynamics is trivial and solely dynamics produces a glassy behavior) to the problem of glass formation. However, it is reasonable that for the relaxation dynamics in complex landscapes with a distribution of dynamical paths that includes at the same time frequent relaxation channels involving the hopping of large barriers and rare paths characterized by the smallest possible barriers. In this case, the large dimensionality of the configurational space can play the two previously discussed roles at the same time. In this Rapid Communication we analyze the problem of activated dynamics in a multidimensional configurational space from this particular perspective. We mainly focus on the out-of-equilibrium and aging properties of the dynamics. We discuss a model in which an entropy of paths (originated by the frequency of possible relaxation processes) and the activation energy compete during the dynamics, selecting the fastest relaxation channels among the more frequent but activated ones, despite the presence of nonactivated alternatives. In this case, the contribution of the entropy of paths surprisingly gives rise, in a long-time coarse-grained description, to a genuine out-of-equilibrium relaxation behavior typical of standard activated dynamics (trap models). This result points in the direction of an intriguing reconciliation between the aforementioned, and up to now fully competing, approaches of the RFOT thermodynamic picture and the nonactivated purely dynamical approach (purely entropic in nature) to glassy behavior, since we show that in the step model both entropic and energetic effects are at work. 
The model. Our system of interest can be in $M$ configurations $i$, with energy $E_{i}<0$ given by independent identically distributed random variables extracted from an exponential distribution $\rho(E)=\lambda \exp (\lambda E) \theta(-E)$. We introduce a Metropolis dynamics with nonzero transition rates from any level $i$ to any other level $j$ given by

$$
w_{i, j}=\left\{\begin{array}{ll}
1 & \text { if } E_{i} \geqslant E_{j} \\
\exp \left[-\beta\left(E_{j}-E_{i}\right)\right] & \text { if } E_{i}<E_{j}
\end{array} .\right.
$$

This model only depends on the ratio $\beta / \lambda$, it is equivalent to the one defined by Mezard and Barrat [22,23] and it is called the step model. They used a Glauber dynamics instead of our Metropolis rule.

Step models were introduced in antithesis to preexisting trap models. The latter were first used by Bouchaud [3] as the paradigm of low-temperature-activated dynamics in a multiminima PEL. In the trap case one considers the same exponential distribution $\rho(E)$, but each configuration $i$ occupies the bottom of a trap ideally surrounded by energy barriers $\Delta_{i}=-E_{i}>0$ in any direction. The transition rates read $w_{i, j}=\frac{1}{M} \exp \left(-\beta \Delta_{i}\right)$ and the time for escaping from each configuration $\tau_{i} \propto 1 / w_{i, j} \propto \exp \left(\beta \Delta_{i}\right)$ simply follows an Arrhenius law. At variance, step models were designed without the explicit introduction of potential energy barriers to study the influence of the entropy of possible relaxation paths in the context of a nonactivated slow dynamics of glassy systems.

Here, we focus on step models to study the contribution of the entropy of paths together with activated relaxation, as it should be for deeply supercooled liquids. Each dynamical move is, in fact, decided by the competition between a possible energy increase, favored by an exponentially large number of high-energy configurations and hampered by a low acceptance rate, and an exponentially small number of descending paths with high acceptance rate. The entropic and thermal dynamical drives are quantified, respectively, by $\lambda$ and $\beta=1 / T$. The study of step models is also motivated by the recent interest $[6,8]$ in a possible generalization of the trap-like aging phenomenology to models with more realistic dynamical rules, as, for example, the Metropolis one.

First studies of the step model [22] (and recently [23]) found that its low-temperature slow relaxation only follows nonactivated dynamical paths. A first evidence of an activated dynamical regime at intermediate temperatures was presented in Ref. [24]. However, even in the regime where thermal activation sets in, typical sojourn times in single configurations were found to be always determined by entropic mechanisms [24]. An explicit thermal activation was reintroduced in the dynamics as the best means to recover the low-temperature activation features of more realistic systems [24]. Let us summarize. We start from $T=0$ and increase $T$. We are in an entropic phase, which we will call the low-temperature regime. When $\beta$, decreasing, reaches the value $2 \lambda$, we enter, as we will see, an important regime where the behavior of the system is decided both by entropy and by energy; we call this phase an intermediate $T$ regime. When eventually $\beta$ reaches the value $\lambda$ we enter a high- $T$ regime where the system easily reaches equilibrium.

Here we ignore the fully entropic low-temperature range, and we avoid the explicit introduction of thermal activation.

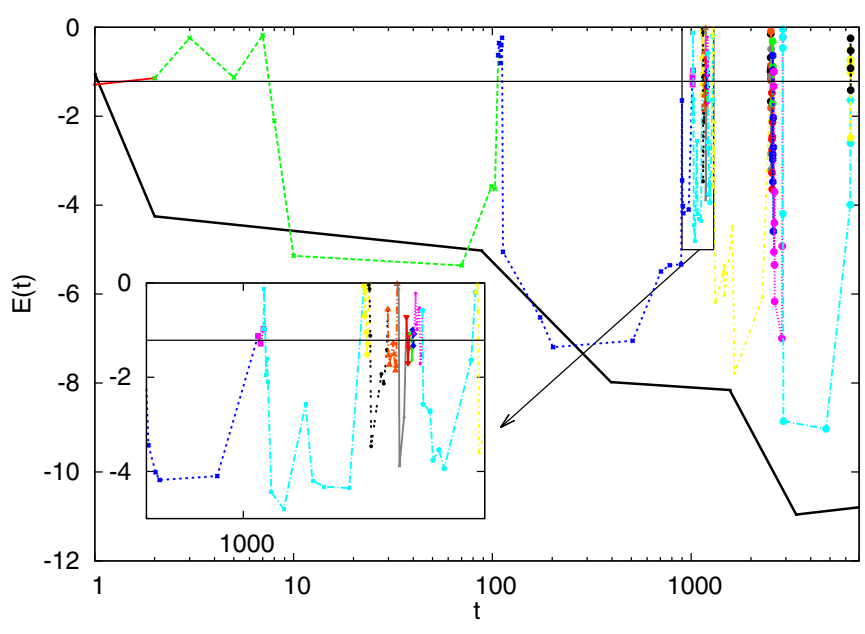

FIG. 1. (Color online) Two time series (and a zoom of one of them in the inset) of the energy explored by step models for $\rho(E)$ with $\lambda=1$. The continuous line is for $T=0.25$. Broken lines are for $T=0.75$. The horizontal line marks the level of the threshold energy $E_{\mathrm{th}}$ for $T=0.75$, when it can be defined. Different types of broken lines are for different basins, according to the definition in the text.

We focus instead on the intermediate temperature regime to provide a paradigm and an explanation for the spontaneous formation of activation barriers during the dynamics, as a pure result of the competition between entropic and energetic dynamical drives. As it was suggested by previous intriguing results [24], these barriers do not directly give rise to usual thermal activation. Yet, in our study we are able to show that in this entropy-energy-ruled dynamical regime an effective traplike out-of-equilibrium phenomenology typical of models with genuine thermal activation unexpectedly emerges.

Two dynamical regimes in step models. We study the $M=$ $\infty$ case, where the system can access an infinite number of energy levels. At each step, we start from a configuration with energy $E_{i}$ and we propose as a trial energy $E_{j}$, a random variable extracted from the distribution $\rho(E)$. If the Metropolis move is accepted, with rate $w_{i, j}, E_{j}$ becomes the new energy of the system, otherwise the energy $E_{j}$ is disregarded, and the old energy $E_{i}$ is kept. Time always increases by one unit.

Numerical simulations show that the model switches, when $T$ changes, between two completely different behaviors. For low or zero temperatures we observe the entropy-ruled regime studied by Barrat and Mezard [22,23]. The level of the explored energies continuously decreases as $t$ increases (see the continuous thick line in Fig. 1). For low energies the trapping time in each configuration becomes larger and larger since moves that increase the energy are rarely accepted, and moves that lower the energy are only seldom proposed.

At intermediate temperatures something completely different occurs. As shown by the broken line in Fig. 1 (the dash code of its segments will be discussed later), the energy episodically returns to high values. This phenomenon signals the spontaneous emergence of effective barriers that the system has to overcome during the dynamics. Dynamics is fast at high energies but, at large $t$, deeper and deeper configurations are progressively explored arresting the dynamics for increasingly long times. 


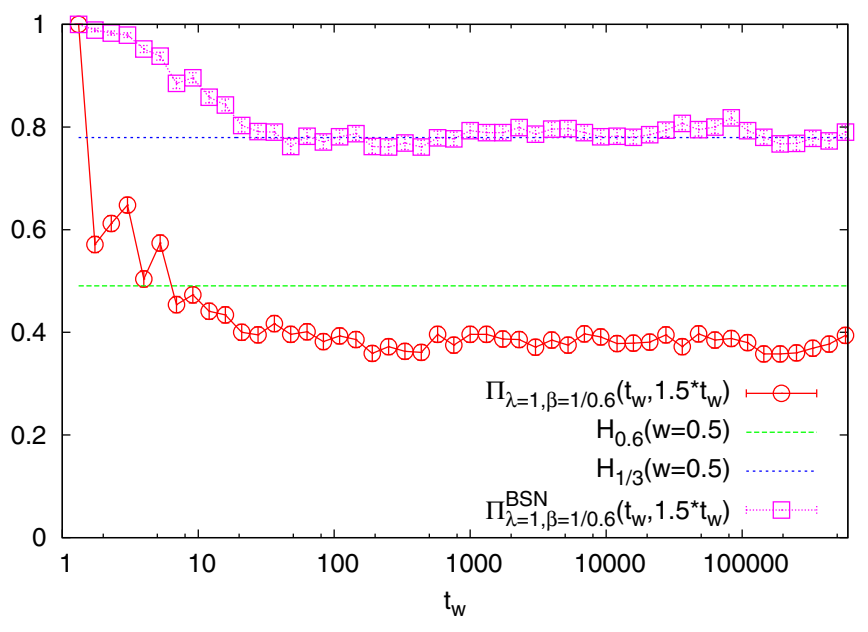

FIG. 2. (Color online) The probability of not changing configuration between $t_{w}$ and $t_{w}+0.5 t_{w}, \Pi_{\lambda=1, \beta=1 / 0.6}\left(t_{w}, t_{w}+0.5 t_{w}\right)$, does not converge, for large $t_{w}$, to the naive expectation from trap paradigm, $H_{0.6}(w=0.5)$ (lower line). Instead, the probability of not changing basin between the same times $\Pi_{\lambda=1, \beta=1 / 0.6}^{B S N}\left(t_{w}, t_{w}+0.5 t_{w}\right)$ converges remarkably well in the large $t_{w}$ limit to the equivalent trap model result $H_{2-1 / 0.6}(w=0.5)$ (upper line).

Bertin found first indirect evidences [24] of a thermally activated behavior in this second regime by observing the large $t / t_{w}$ power-law decay of correlation functions between $t_{w}$ and $t_{w}+t$. However, we found that a clear discrepancy emerges comparing the numerical results of the probability of not changing configuration between $t_{w}$ and $t+t_{w}, \Pi_{\lambda, \beta}\left(t_{w}, t_{w}+t\right)$, and its large $t_{w}$ limit $C_{\lambda, \beta}(w)=$ $\lim _{t_{w} \rightarrow \infty ; t / t_{w}=w} \Pi_{\lambda, \beta}\left(t_{w}, t_{w}+t\right)$ in the step model to the theoretical expectations for trap models [3-6]. In trap models for any choice of parameters $\lambda, \beta[4,5]$ one has

$$
C_{\lambda, \beta}(w)=H_{\lambda / \beta}(w)
$$

where

$$
H_{x}(w) \equiv \frac{\sin (\pi x)}{\pi} \int_{w}^{\infty} d u \frac{1}{(1+u) u^{x}} .
$$

As we show in Fig. 2, $C_{\lambda, \beta}(w)$ for step models has a finite and well-defined value but it is different from $H_{\lambda / \beta}(w)$.

The main feature that determines the aging behavior of the trap models [3] is the fat tail power-law distribution of trapping times $p(\tau) \sim \tau^{-(1+\mu)}$ (with $0<\mu<1$ ), which does not change with the observation time $t$ and whose exponent $\mu=\lambda / \beta$ controls the parameter of $H_{x}(w): x=\mu$. In step models, the Metropolis dynamical rule gives rise to a typical distribution of trapping times, which ages during the dynamics [22-24] but which at $t \gg \tau$ also behaves like $p(\tau) \sim \tau^{1+\mu}$, with $\mu=2-\beta / \lambda$. Hence, one could also expect an effective trap model behavior described by an $H_{x}(w)$ with parameter $x=2-\beta / \lambda$. Figure 2 shows that numerical data are in contradiction with this expectation, too. Moreover, if we try to extract empirically the effective parameter $x$ assuming that $C_{\lambda, \beta}(w)=H_{x}(w)$, we find different results for different values of $w$. In conclusion, the trap paradigm apparently does not apply to the $C_{\lambda, \beta}(w)$ obtained for step models.
This analysis confirms the deep difference between step and trap models, besides the emergence of dynamical effective barriers in the first case. As a confirmation of this result let us focus on an estimate of trapping times in the step model. For every energy level $E$, the probability of going upward $P_{\uparrow}(E)$ and downward $P_{\downarrow}(E)$ are

$$
\begin{aligned}
P_{\uparrow}(E) & =\int_{E}^{0} d E^{\prime} \rho\left(E^{\prime}\right) \exp \left[-\beta\left(E^{\prime}-E\right)\right] \\
& =\frac{\lambda}{\beta-\lambda}[\exp (\lambda E)-\exp (\beta E)],
\end{aligned}
$$

and

$$
P_{\downarrow}(E)=\int_{-\infty}^{E} d E^{\prime} \rho\left(E^{\prime}\right)=\exp (\lambda E) .
$$

Hence, in the low-temperature range, where $\beta>\lambda$, at the leading order the trapping time in each configuration $\tau(E)=$ $\frac{1}{p_{\uparrow}(E)+p_{\downarrow}(E)} \sim \exp (-\lambda E)$ does not depend on the temperature [24], as it would for thermal activated relaxation channels.

A coarse-grained description of the Metropolis dynamics. To make a step forward in the study of this anomalous activated regime, also inspired by Refs. [25-27], we analyze the dynamics of the model from a different point of view. We start by focusing on the large dynamical basins that periodically appear in the microscopic time sequence of the explored energy levels. These basins stem from the fact that for $\lambda / \beta<0.5$ or for high $E$ one has $p_{\uparrow}(E)<p_{\downarrow}(E)$, while for $0.5<\lambda / \beta<1$ (which coincides with Bertin's thermal activated regime [24]) and $E<E_{t h} \equiv \frac{1}{\beta-\lambda} \ln \left(\frac{2 \lambda-\beta}{\lambda}\right)$, the opposite inequality holds: $p_{\uparrow}(E)>p_{\downarrow}(E)$ [28]. This means that if the dynamics goes below $E_{\mathrm{th}}$ (represented in Fig. 1 by the thin horizontal line) it will be driven back to larger energy levels $E>E_{\text {th }}$ before falling again deep down in the PEL. This threshold energy plays the role of a dynamical potential energy barrier self-generated by the competition between rare downward descents and exponentially suppressed Metropolis transitions toward higher energies. We will therefore use $E_{\text {th }}$ to define spontaneously forming dynamical basins. Each time $E$ crosses $E_{\text {th }}$ from below, we say that the system changes basin (and we change the line-style in Fig. 1). Within this construction in terms of dynamical basins we can now study the probability distribution function of the trapping time in each basin $p\left(\tau^{\mathrm{BSN}}\right)$, for $t \gg \tau^{\mathrm{BSN}}$. Not surprisingly, the trapping times distribution changes when we consider basins instead of configurations, but the power-law behavior for large $\tau$ is described by the same exponent $1+2-\beta / \lambda$ as before. This power law behavior of $p\left(\tau^{\mathrm{BSN}}\right)$ has been already shown for a generalization of the step model in the context of the on-off intermittency problem [29]. Most importantly, in terms of the spontaneously formed dynamical basins, we can also define a new correlation function $\Pi_{\lambda, \beta}^{\mathrm{BSN}}\left(t_{w}, t_{w}+t\right)$ as the probability of not changing basin between $t_{w}$ and $t_{w}+t$ and study its large $t_{w}$ limit, $C_{\lambda, \beta}^{\mathrm{BSN}}\left(t / t_{w}=w\right)$. In this case a very good agreement is found between $C_{\lambda, \beta}^{\mathrm{BSN}}\left(t / t_{w}=w\right)$ and $H_{2-\beta / \lambda}(w)$ as it is shown in Fig. 2. We hence report directly the results for the $p\left(\tau^{\mathrm{BSN}}\right)$ exponent in Fig. 3 that at last perfectly overlap with the $x$ parameter extracted from $C_{\lambda, \beta}^{\mathrm{BSN}}\left(t / t_{w}=w\right)$ at different values of $w$ and $\beta$. 


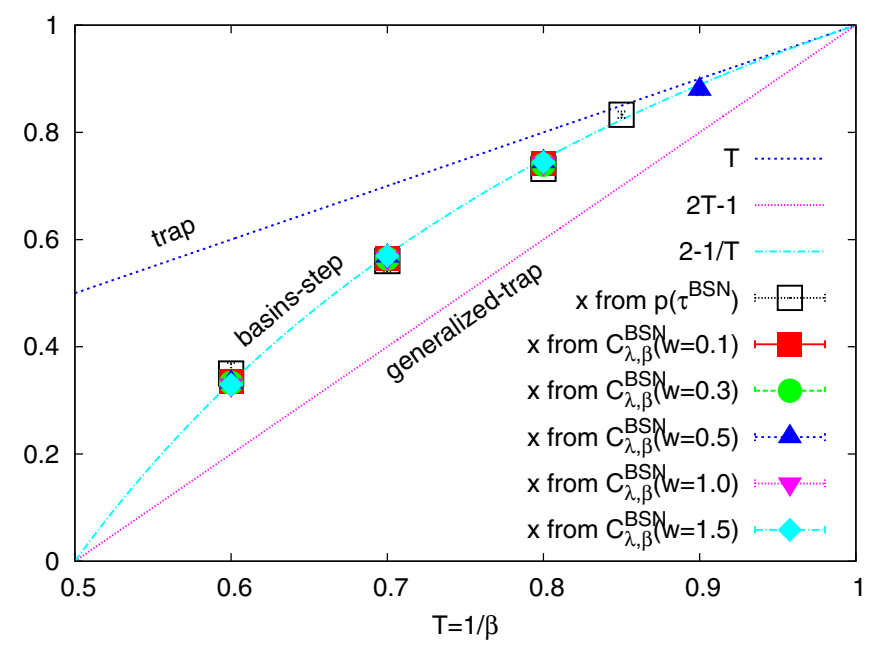

FIG. 3. (Color online) The aging parameter $x$ (obtained from the exponent of the power-law tail of $p\left(\tau^{\mathrm{BSN}}\right)$, empty squares, and from the solution of the $H_{x}(w)=C_{\lambda, \beta}^{\mathrm{BSN}}(w)$ inverse problem, filled symbols) is reported for a step model with $\lambda=1$ as a function of the temperature. We also show the expectation for $x$ in simple trap models (upper straight line), generalized trap models (middle curve), and step models (lower straight line).

In practice the dynamical behavior in terms of a basins description of a step model with $\beta \in[\lambda, 2 \lambda)$ maps onto usual aging of trap models with $\beta_{T} \in[\lambda, \infty)$ if their aging parameters $x$ correspond: $\lambda / \beta_{T}=2-\beta / \lambda$. We observe this on the correlation function $\Pi$ that qualifies the dynamical behavior of the system.

In Fig. 3 we compare the parameter $x$ with the expectation coming from a naive correspondence to trap models, $x_{T}=$ $\lambda / \beta$, and with the aging parameter $x_{\mathrm{GT}}$ for generalized trap models where transition rates depend at each step on initial and final configurations with the same weight: $w_{i, j}(a)=$ $\exp \left[-\beta a E_{i}+\beta(1-a) E_{j}\right][5,30-32]$ with $a=0.5$. In this case, one can show [7] that $x_{\mathrm{GT}}=(\lambda / \beta-a) /(1-a)$ (see Ref. [33] for a discussion of the physical meaning of this result), hence $x_{\mathrm{GT}}=2 \lambda / \beta-1$ for $a=0.5$. The parameter $x$ obtained in step models differs only by a factor $\lambda / \beta$ from $x_{\mathrm{GT}}$. Once more, this result highlights the difference between the entropy-ruled activation of step models [where trapping time is $\tau \sim \exp (-\lambda E)]$ and the thermal activation of trap models [where $\tau \sim \exp (-\beta E)$ ] and excludes any qualitative difference in the long-time dynamical behavior.

Discussion and conclusion. We have studied the out-ofequilibrium dynamics of a simple model with rough PEL called step model. In step models, potential energy barriers are not introduced a priori, as opposed to trap models where explicit thermal activated relaxation mechanisms occur. Nevertheless, in an intermediate temperature regime $0.5<\lambda / \beta<1$, a nontrivial interaction between entropy and energy of typical relaxation paths takes place. As a result, the dynamics shows the presence of deep basins separated by barriers that form spontaneously, reaching a threshold energy $E_{\text {th }} . E_{\text {th }}$ directly stems from the entropy trapping mechanism: the larger the rate $\lambda$ of the energy distribution, the less escaping directions with low-energy activation cost are available, and the larger is the resulting threshold energy $E_{\mathrm{th}}$. Moreover the trapping times in configurations, $\tau$, and basins, $\tau^{\mathrm{BSN}}$, are entirely determined by the entropy of escaping directions. In spite of that, the typical phenomenology of the genuine trap paradigm, classically referred to thermal activation, can be recognized in the aging behavior of this model provided a description of the dynamics in terms of the spontaneously-formed basins is adopted.

The trap-like aging behavior is hence extended by this study to an entropy-energy ruled dynamics like the one of step models. The introduced coarse-grained basin description of the dynamics plays a major role in this analysis. It does not change the power-law tail behavior of the distribution function of the typical trapping times, a necessary condition for trap model-like aging. On the other hand, it takes advantage of the existence of a reference threshold energy to transform the step-model dynamics into a renewal process [4]: each time the threshold energy is reached, the dynamics loses memory of the past, hence subsequent basins are always mutually independent. This was the missing link for establishing the trap model aging phenomenology in the step model.

The step model provides an entropy-energy-ruled relaxation process that could potentially characterize slow dynamics in more realistic glassy systems. Also in these cases, relaxation in the multidimensional configurational space could be controlled by a competition between the energy of the typical barriers and the entropy of the possible dynamical paths. The same competition could determine a specific threshold energy for relaxation processes (the importance of a threshold energy for glassy dynamics was firstly pointed out in Ref. [34]), transforming generic out-of-equilibrium dynamics into a renewal process. In this context, the basin description that we have introduced would be a fundamental analytical tool to recognize the possible presence of an underlying trap-model-like aging behavior.

Acknowledgments. We are grateful to Gerard Ben Arous, Eric Bertin, Giulio Biroli, Jean-Philippe Bouchaud, Jorge Kurchan, and Gilles Tarjus for interesting remarks and discussions. We acknowledge support from the ERC grant CRIPHERASY (Grant No. 247328).
[1] M. Ediger, C. Angell, and S. R. Nagel, J. Phys. Chem. 100, 13200 (1996).

[2] M. Goldstein, J. Chem. Phys. 51, 3728 (1969).

[3] J.-P. Bouchaud, J. Phys. I 2, 1705 (1992).

[4] J.-P. Bouchaud and D. S. Dean, J. Phys. I 5, 265 (1995).

[5] G. Ben Arous and J. Cerný, Dynamics of trap models, École d'Été de Physique des Houches LXXXIII "Mathematical Statistical Physics” (North-Holland, Amsterdam, 2006), p. 331.
[6] G. Ben Arous, A. Bovier, and V. Gayrard, Phys. Rev. Lett. 88, 087201 (2002).

[7] V. Gayrard, arXiv:1008.3855 (2010).

[8] V. Gayrard, arXiv:1402.0388 (2014).

[9] L. R. Fontes, M. Isopi, C. M. Newman, and D. L. Stein, Phys. Rev. Lett. 87, 110201 (2001).

[10] W. Götze and L. Sjogren, Rep. Prog. Phys. 55, 241 (1992). 
[11] G. H. Fredrickson and H. C. Andersen, Phys. Rev. Lett. 53, 1244 (1984).

[12] D. Chandler and J. P. Garrahan, Annu. Rev. Phys. Chem. 61, 191 (2010).

[13] T. R. Kirkpatrick, D. Thirumalai, and P. G. Wolynes, Phys. Rev. A 40, 1045 (1989).

[14] A. Cavagna, Phys. Rep. 476, 51 (2009).

[15] L. Berthier and G. Biroli, Rev. Mod. Phys. 83, 587 (2011).

[16] G. Parisi and F. Zamponi, Rev. Mod. Phys. 82, 789 (2010).

[17] P. Charbonneau, J. Kurchan, G. Parisi, P. Urbani, and F. Zamponi, Nature Commun. 5, 3725 (2014).

[18] J. Kurchan, Glasses and grains: Poincaré seminar 2009 (Springer, Berlin, 2011), Chap. 1.

[19] J. Kurchan and L. Laloux, J. Phys. A 29, 1929 (1996).

[20] C. Toninelli and G. Biroli, J. Stat. Phys. 126, 731 (2007).

[21] J. Olejarz, P. L. Krapivsky, and S. Redner, Phys. Rev. E 83, 030104 (2011).

[22] A. Barrat and M. Mézard, J. Phys. I 5, 941 (1995).

[23] E. Bertin, J. Phys. A 43, 345002 (2010).
[24] E. M. Bertin, J. Phys. A 36, 10683 (2003).

[25] R. A. Denny, D. R. Reichman, and J.-P. Bouchaud, Phys. Rev. Lett. 90, 025503 (2003).

[26] B. Doliwa and A. Heuer, Phys. Rev. E 67, 030501 (2003); 67, 031506 (2003).

[27] A. Heuer, J. Phys. Condens. Matter 20, 373101 (2008).

[28] Despite inessential changes on the value of $E_{\mathrm{th}}$, other choices for $\rho(E)$ (i.e., Gaussian) are expected to give rise to the same dynamical behavior as soon as the probability of finding configurations at lower energy is exponentially suppressed.

[29] E. Bertin, Phys. Rev. E 85, 042104 (2012).

[30] B. Rinn, P. Maass, and J.-P. Bouchaud, Phys. Rev. Lett. 84, 5403 (2000)

[31] B. Rinn, P. Maass, and J.-P. Bouchaud, Phys. Rev. B 64, 104417 (2001).

[32] C. Monthus, Phys. Rev. E 68, 036114 (2003).

[33] C. Cammarota and E. Marinari (unpublished).

[34] L. F. Cugliandolo and J. Kurchan, Phys. Rev. Lett. 71, 173 (1993). 